\title{
Optimization of Residual Wall Thickness Uniformity in Short-Fiber-Reinforced Composites Water-Assisted Injection Molding Using Response Surface Methodology and Artificial Neural Network-Genetic Algorithm
}

\author{
Haiying Zhou $\mathbb{D}^{1,2}$ Hesheng Liu ${ }^{1}{ }^{1,2}$ Tangqing Kuang, ${ }^{3}$ Qingsong Jiang, ${ }^{2}$ Zhixin Chen, \\ and Weiping $\mathrm{Li}^{2}$ \\ ${ }^{1}$ School of Mechanical and Electrical Engineering, Nanchang University, Nanchang 330031, China \\ ${ }^{2}$ Jiangxi Province Key Laboratory of Polymer Micro/Nano Manufacturing and Devices, East China University of Technology, \\ Nanchang 330013, China \\ ${ }^{3}$ School of Mechanical and Electrical Engineering, East China Jiaotong University, Nanchang 330013, China
}

Correspondence should be addressed to Hesheng Liu; hsliu@vip.163.com

Received 17 June 2019; Accepted 29 July 2019; Published 26 February 2020

Guest Editor: Yun Zhang

Copyright $\odot 2020$ Haiying Zhou et al. This is an open access article distributed under the Creative Commons Attribution License, which permits unrestricted use, distribution, and reproduction in any medium, provided the original work is properly cited.

\begin{abstract}
This study aimed at improving the residual wall thickness uniformity (RWTU), which was closely related to the mechanical properties of plastic parts with a hollow cross-section, in short-fiber reinforced composites (SFRC) overflow water-assisted injection molding (OWAIM). The influences of five independent process parameters (melt temperature, mold temperature, delay time, water pressure, and water temperature) on RWTU were investigated through the methods such as central composite design, regression equation, and analyses of variance. Response surface methodology (RSM) and artificial neural network (ANN) optimized by genetic algorithm (GA) were employed to map the relationship between the process parameters and the standard deviation (SD) depicting the RWTU. Comparison assessments of three models (RSM, ANN, and ANN-GA) were carried out through some statistical indexes. It was concluded that the effect of melt temperature, delay time, and water temperature were significant to RWTU; the hybrid ANN-GA model had the best performance for predicting SD compared with RSM and ANN; the least SD obtained in optimization using ANN-GA as a fitness function was 0.0972 .
\end{abstract}

\section{Introduction}

Overflow water-assisted injection molding (OWAIM) is a promising method for producing functional plastic parts with hollow sections and thin residual wall thickness (RWT) [1-3]. The process of OWAIM includes two steps. First, the functional part cavity is filled with the melted polymer. Second, after a short delay time, the high-pressure water is injected into the core to push the melt into the overflow cavity to form a functional part with a hollow cross-section. This technology has many advantages such as polymer saving, short cycle time, lower injection pressure, less warpage, better surface quality, enhanced flexibility in mold design, etc., [4].

Great attention has been paid to the RWT which is an important indicator for assessing the quality of overflow water-assisted injection molded parts [5-9]. The experiments and simulations indicated that the distribution of the RWT was uneven in OWAIM. The plastic parts with thin, uneven RWT are difficult to meet the mechanical performance requirement in special application. Using short-fiber reinforced composites (SFRC) as raw materials can significantly improve the mechanical properties of a plastic part $[10,11]$. But it makes the process of OWAIM more complicated which results in a more uneven RWT. The residual wall thickness uniformity (RWTU) of a plastic part is related to the overall mechanical properties. Thus, for the wide application in different fields, it is urgent to improve the RWTU of plastic parts in OWAIM.

In the traditional plastic manufacturing industry, product quality improvement mainly depends on the workers' experience and trial and error, which is costly, time-consuming, and greatly 
decrease the product competitiveness [12]. Fortunately, the development of information processing technology, using statistical methods, and artificial intelligence algorithm for modeling and optimizing quality objectives, significantly shorten the cycle time of product designing and reduce the product cost [13-15]. RWTU is influenced by the many factors such as material properties, mold structure, process parameter, etc. Generally, adjusting the process parameter setting for optimization is an approach adopted by industry plants. Thus, it is crucial to construct the relationships between the process parameters and RWTU.

Response surface methodology (RSM), based on statistical theory, is a classic and effective approach and has been widely applied for modeling and optimization. RSM is very useful for developing, improving, and optimizing the responses that are affected by multiple independent variables $[16,17]$. RSM can be applied to evaluate the correlation between the responses and the independent variables and define the influences of the independent variables individually or in combination. However, the nonlinearity of OWAIM processes may be difficult for RSM to achieve better model accuracy and generalization. Artificial neural network (ANN), as an effective method for mapping linear and nonlinear relationships between factors and targets, is widely used for modeling, prediction, classification, and pattern recognition $[18,19]$. However, the performance of ANN trained by the gradient decent algorithm is greatly affected using the inappropriate initial weights and bias, which causes local minima [20]. Genetic algorithm (GA), inspired by the biological evolution theory, is a global optimization tool and can be used to search the optimal initial weights and bias for ANN. The combination of ANN and GA (ANN-GA) has been successfully used in optimization studies $[21,22]$.

Up to our knowledge, limited worthwhile research has been implemented for the improvement of RWTU in SFRC OWAIM. In this study, the numerical experiments, arranged using a central composite design (CCD), have been carried out. RSM and ANN-GA were employed to map the relationship between the RWTU and the process parameters (melt temperature, mold temperature, delay time, water pressure, and water temperature). The significance of five process parameters was studied through the analyses of variance (ANOVA). The prediction performance of RSM, ANN, and ANN-GA models were compared using the linear regression equation and statistical indicators. Finally, the model with the best prediction performance was used as the fitness function of the GA to optimize the RWTU of the plastic part in SFRC OWAIM.

\section{Methods}

2.1. Related Mathematical Model. During the simulation of OWAIM, the melt flow is regarded as nonisothermal, transient, and nonNewtonian. It is assumed that the melt is incompressible, laminar, and the inertia term is ignored. The basic governing equations for melt flow are as follows.

$$
\begin{gathered}
\frac{\partial P}{\partial t}+\nabla \cdot \rho \boldsymbol{u}=0 \\
\frac{\partial}{\partial t}(\rho \boldsymbol{u})+\nabla \cdot(\rho \boldsymbol{u} \boldsymbol{u}-\boldsymbol{\tau})=\rho g
\end{gathered}
$$

$$
\begin{gathered}
\rho C_{p}\left(\frac{\partial T}{\partial t}+\boldsymbol{u} \cdot \nabla T\right)=\nabla \cdot(k \nabla T)+\eta \gamma^{2}, \\
\boldsymbol{\tau}=-p \boldsymbol{I}+\eta\left(\nabla \boldsymbol{u}+\nabla \boldsymbol{u}^{T}\right),
\end{gathered}
$$

where $P$ is the pressure; $T$ the temperature; $t$ the time; $\boldsymbol{u}$ the speed; $\boldsymbol{\tau}$ the stress tensor; $\rho$ the density; $\eta$ the viscosity; $k$ the thermal conductivity; $C_{p}$ the specific heat; and $\gamma$ the shear strain.

A constitutive equation with seven parameters is used to describe the relationship between melt viscosity and temperature and shear rate.

$$
\begin{gathered}
\eta(\dot{\gamma}, T, P)=\frac{\eta_{0}(T, P)}{1+\left(\eta_{0} \dot{\gamma} / \tau^{*}\right)^{1-n}}, \\
\eta_{0}(T, P)=D_{1} \exp \left(\frac{-A_{1}\left(T-T_{c}\right)}{A_{2}+\left(T-T_{c}\right)}\right), \\
T_{c}=D_{2}+D_{3} P, \\
A_{2}=\widetilde{A}_{2}+D_{3} P,
\end{gathered}
$$

where $\eta$ is the viscosity; $\eta_{0}$ the zero shear viscosity; $\dot{\gamma}$ the shear rate; $\tau^{*}$ the material constant; $n$ the power rate index; $T$ the melt temperature; $T_{c}$ the glass transition temperature; $D_{1}, D_{2}$, $D_{3}, A_{1}$, and $\widetilde{A_{2}}$ are the relative constants associated with the selected material.

In the high-pressure water filling stage, the volume of fluid (VOF) model is used to track the interface of the melt and water. $F_{i}$ is the volume fraction of the $i$-th phase, and $0 \leq F_{i} \leq$ 1. When a unit is completely occupied by the $i$-th phase, $F_{i}$ takes a value of 1 . When a unit does not have the $i$-th phase, $F_{i}$ takes a value of zero.

$$
\frac{\partial F_{i}}{\partial t}+\nabla \cdot\left(\boldsymbol{u} F_{i}\right)=0
$$

2.2. Geometric Model in Simulation. As shown in Figure 1, the geometric model used in the simulation was composed of a runner, an overflow cavity and a functional plastic part with a diameter $16 \mathrm{~mm}$ and two elbows. The model built by Pro/E was meshed using the commercial software of Moldex3D. The numbers of mesh nodes and mesh elements were 60175 and 195118, respectively. The short glass fiber reinforced PP (Fiberfil J-68/20/E with a short fiber mass fraction of $20 \%$ and an aspect ratio of 20) was selected as the raw material in the simulation and its properties were available in the data bank of Moldex3D.

2.3. Definition of Residual Wall Thickness Uniformity. The values of RWT were measured at ten different locations along the central axis of the functional part as shown in Figure 2. Standard deviation (SD), which reflects the degree of dispersion among individuals in a group, was defined as an indicator for evaluating the RWTU. The formula of SD can be expressed as the following: 


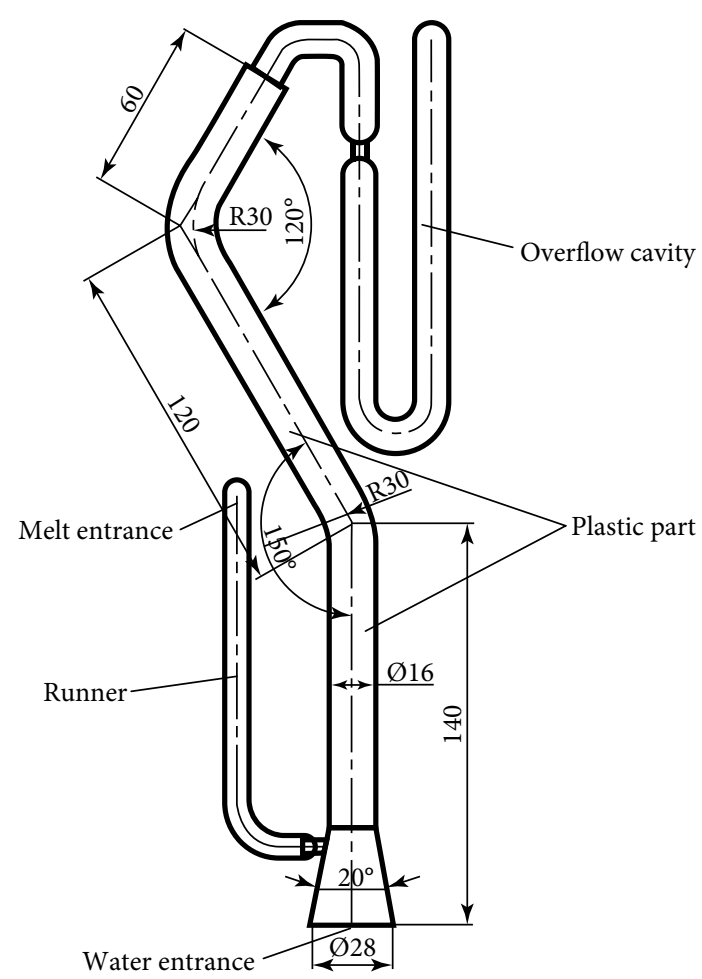

Figure 1: Geometric model for simulation.

$$
\mathrm{SD}=\sqrt{\frac{1}{N} \sum_{i=1}^{N}\left(R_{i}-R_{\mathrm{avg}}\right)^{2}}
$$

where $R_{i}$ is the value of RWT measured at the $i$-th point; $R_{\mathrm{avg}}$ is the average value of RWT; $N$ is the total number of points.

2.4. Experimental Design. The process parameters such as melt temperature, mold temperature, delay time, water pressure, and water temperature were considered in this study. In general, those process parameters were easily controlled in the experiments and production for adjusting the important indicators of plastic parts. The process windows recommended for OWAIM were melt temperature $\left[210^{\circ} \mathrm{C}, 230^{\circ} \mathrm{C}\right]$, mold temperature $\left[42^{\circ} \mathrm{C}, 62^{\circ} \mathrm{C}\right]$, delay time $[1 \mathrm{~s}, 5 \mathrm{~s}]$, water pressure $[8 \mathrm{MPa}, 12 \mathrm{MPa}]$, and water temperature $\left[20^{\circ} \mathrm{C}, 30^{\circ} \mathrm{C}\right]$. In order to reduce the experiment times and comprehensively examine the influences of the process parameters on RWTU, the CCD based on RSM was applied to arrange the simulation experiments. The coded and actual values of five independent process parameters are shown in Table 1. Fifty experiments composed of 42 factorial and axial points and 8 center points are demanded for the CCD with three levels and five factors. The center points with the same process parameters result in the same RWT in the simulations. Therefore, the total number of experiments is 43 . The details of the arrangements are revealed in Table 2.

2.5. Response Surface Methodology. The method of RSM based on the statistical technology was employed for the multiple regression analysis of experimental data obtained from the CCD. The relationship between the independent process parameters and the response SD is depicted using a secondorder polynomial equation.

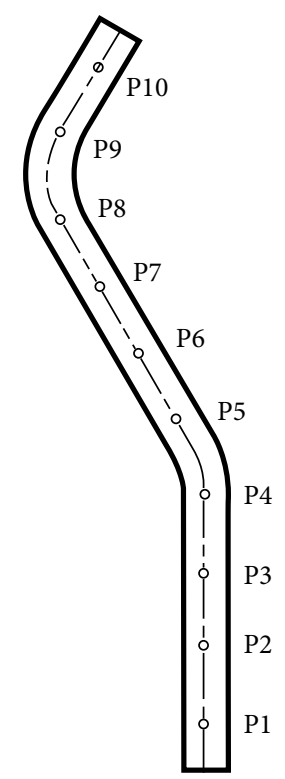

FiguRE 2: Locations for measuring residual wall thickness.

TABLE 1: Factors and level of process parameters.

\begin{tabular}{lccc}
\hline Process parameter & \multicolumn{3}{c}{ Level } \\
& Low & Mean & High \\
\hline Melt temperature $\left(X_{1}\right) /\left[{ }^{\circ} \mathrm{C}\right]$ & $-1(210)$ & $0(220)$ & $1(230)$ \\
Mold temperature $\left(X_{2}\right) /\left[{ }^{\circ} \mathrm{C}\right]$ & $-1(42)$ & $0(52)$ & $1(62)$ \\
Delay time $\left(X_{3}\right) /[\mathrm{s}]$ & $-1(1)$ & $0(3)$ & $1(5)$ \\
Water pressure $\left(X_{4}\right) /[\mathrm{MPa}]$ & $-1(8)$ & $0(10)$ & $1(12)$ \\
Water temperature $\left(X_{5}\right) /\left[{ }^{\circ} \mathrm{C}\right]$ & $-1(20)$ & $0(25)$ & $1(30)$ \\
\hline
\end{tabular}

$$
\mathrm{SD}=\beta_{0}+\sum_{i=1}^{5} \beta_{i} X_{i}+\sum_{i=1}^{4} \sum_{j=i+1}^{5} \beta_{i j} X_{i} X_{j}+\sum_{i=1}^{5} \beta_{i i} X_{i}^{2}
$$

where $X_{i}$ and $X_{j}$ are the independent process parameters; $\beta_{0}$ is the intercept constant; $\beta_{i}$ is the linear coefficient; $\beta_{i j}$ is the interaction coefficient and $\beta_{i i}$ is the quadratic coefficient. The regression studies and ANOVA were implemented using the software of Design-Expert 8.0.

2.6. Artificial Neural Network. ANN inspired by biologic neural system is a computing model used to map linear or nonlinear factors and responses relationships. An ANN model comprises three parts: one input layer, one or more hidden layers, and one output layer. Each layer consists of a number of neurons. The numbers of neurons in the input layer and the output layer are determined by the numbers of factors and responses, respectively. In this study, an ANN model with one hidden layer was employed for modeling. The gradient descent algorithm was used for training model. The transfer functions of "Tansig" and "Purelin" were applied in the hidden layer and the output layer, respectively. By changing the number of neurons in the hidden layer from 5 to 15, the ANN topology of 5-11-1 was determined according to the minimum mean square error between the targets and the outputs. The ANN model is demonstrated in Figure 3. 
TABLE 2: Experimental matrix and corresponding results.

\begin{tabular}{|c|c|c|c|c|c|c|c|c|c|c|c|c|c|}
\hline No. & $X_{1}$ & $X_{2}$ & $X_{3}$ & $X_{4}$ & $X_{5}$ & $\mathrm{SD}$ & No. & $X_{1}$ & $X_{2}$ & $X_{3}$ & $X_{4}$ & $X_{5}$ & $\mathrm{SD}$ \\
\hline 1 & 0 & 0 & -1 & 0 & 0 & 0.177 & 23 & -1 & 1 & -1 & -1 & 1 & 0.180 \\
\hline 2 & -1 & -1 & -1 & 1 & 1 & 0.185 & 24 & 0 & 0 & 0 & 0 & 0 & 0.150 \\
\hline 3 & 1 & -1 & 1 & 1 & 1 & 0.183 & 25 & 1 & -1 & 1 & -1 & -1 & 0.125 \\
\hline 4 & 0 & 0 & 0 & -1 & 0 & 0.164 & 26 & 0 & 0 & 0 & 1 & 0 & 0.163 \\
\hline 5 & -1 & -1 & 1 & -1 & 1 & 0.163 & 27 & 1 & 1 & -1 & 1 & 1 & 0.189 \\
\hline 6 & -1 & -1 & -1 & 1 & -1 & 0.224 & 28 & 1 & -1 & 1 & -1 & 1 & 0.215 \\
\hline 7 & 1 & 1 & 1 & -1 & 1 & 0.239 & 29 & -1 & -1 & 1 & 1 & 1 & 0.16 \\
\hline 8 & 1 & -1 & -1 & 1 & 1 & 0.230 & 30 & -1 & 1 & -1 & 1 & 1 & 0.207 \\
\hline 9 & 1 & -1 & -1 & -1 & 1 & 0.200 & 31 & -1 & 1 & -1 & 1 & -1 & 0.187 \\
\hline 10 & 0 & 0 & 0 & 0 & -1 & 0.162 & 32 & 1 & 0 & 0 & 0 & 0 & 0.190 \\
\hline 11 & 1 & -1 & -1 & -1 & -1 & 0.201 & 33 & 0 & 0 & 1 & 0 & 0 & 0.158 \\
\hline 12 & 1 & 1 & 1 & 1 & -1 & 0.154 & 34 & -1 & 1 & 1 & 1 & 1 & 0.157 \\
\hline 13 & 1 & -1 & -1 & 1 & -1 & 0.214 & 35 & 1 & 1 & -1 & -1 & 1 & 0.231 \\
\hline 14 & -1 & -1 & 1 & -1 & -1 & 0.163 & 36 & 1 & 1 & 1 & 1 & 1 & 0.187 \\
\hline 15 & -1 & 1 & 1 & 1 & -1 & 0.112 & 37 & -1 & 1 & 1 & -1 & 1 & 0.138 \\
\hline 16 & 0 & 0 & 0 & 0 & 1 & 0.157 & 38 & 1 & -1 & 1 & 1 & -1 & 0.154 \\
\hline 17 & -1 & 1 & -1 & -1 & -1 & 0.150 & 39 & -1 & -1 & -1 & -1 & 1 & 0.173 \\
\hline 18 & 0 & -1 & 0 & 0 & 0 & 0.165 & 40 & 0 & 1 & 0 & 0 & 0 & 0.177 \\
\hline 19 & -1 & -1 & 1 & 1 & -1 & 0.148 & 41 & -1 & 0 & 0 & 0 & 0 & 0.130 \\
\hline 20 & 1 & 1 & -1 & -1 & -1 & 0.169 & 42 & 1 & 1 & 1 & -1 & -1 & 0.135 \\
\hline 21 & -1 & -1 & -1 & -1 & -1 & 0.151 & 43 & 1 & 1 & -1 & 1 & -1 & 0.190 \\
\hline 22 & -1 & 1 & 1 & -1 & -1 & 0.167 & & & & & & & \\
\hline
\end{tabular}

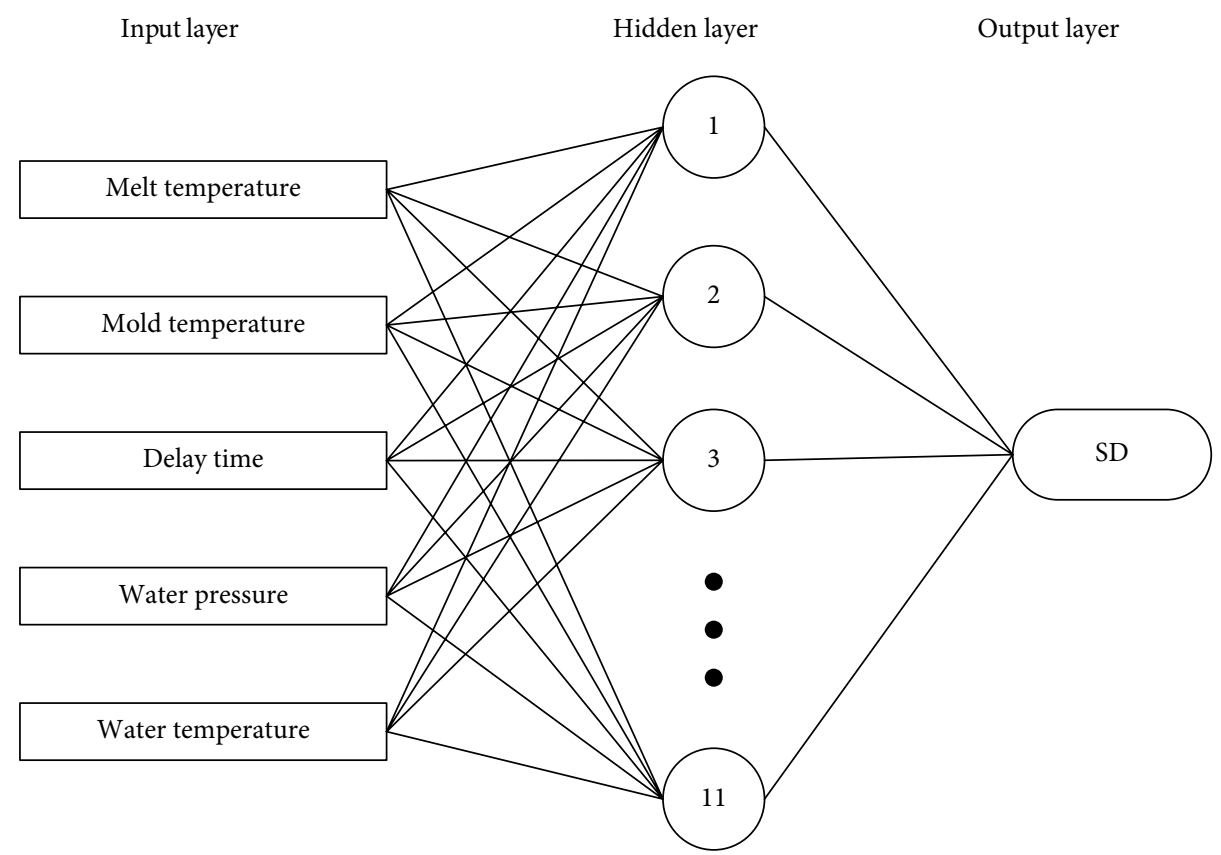

FIGURE 3: Topology of artificial neural network.

2.7. Genetic Algorithm. The genetic algorithm based on natural selection and survival of fitness is a global searching algorithm, and it is widely used in the fields of optimization, pattern recognition, robots, and prediction. Compared with other optimization methods, GA has many advantages including being not easy to be trapped into the local minima, requiring little prior information about the searched objectives, and easy identification of the optima in a complex search space.
The major operations of GA are summarized as follows: (1) Selection: individuals are selected based on their fitness so that better individuals are given a higher chance of being chosen, (2) Crossover: exchange the information of the two parents to generate a new individual according to the crossover probability, (3) Mutation: randomly alter the information of each chromosome according to the mutation probability. 


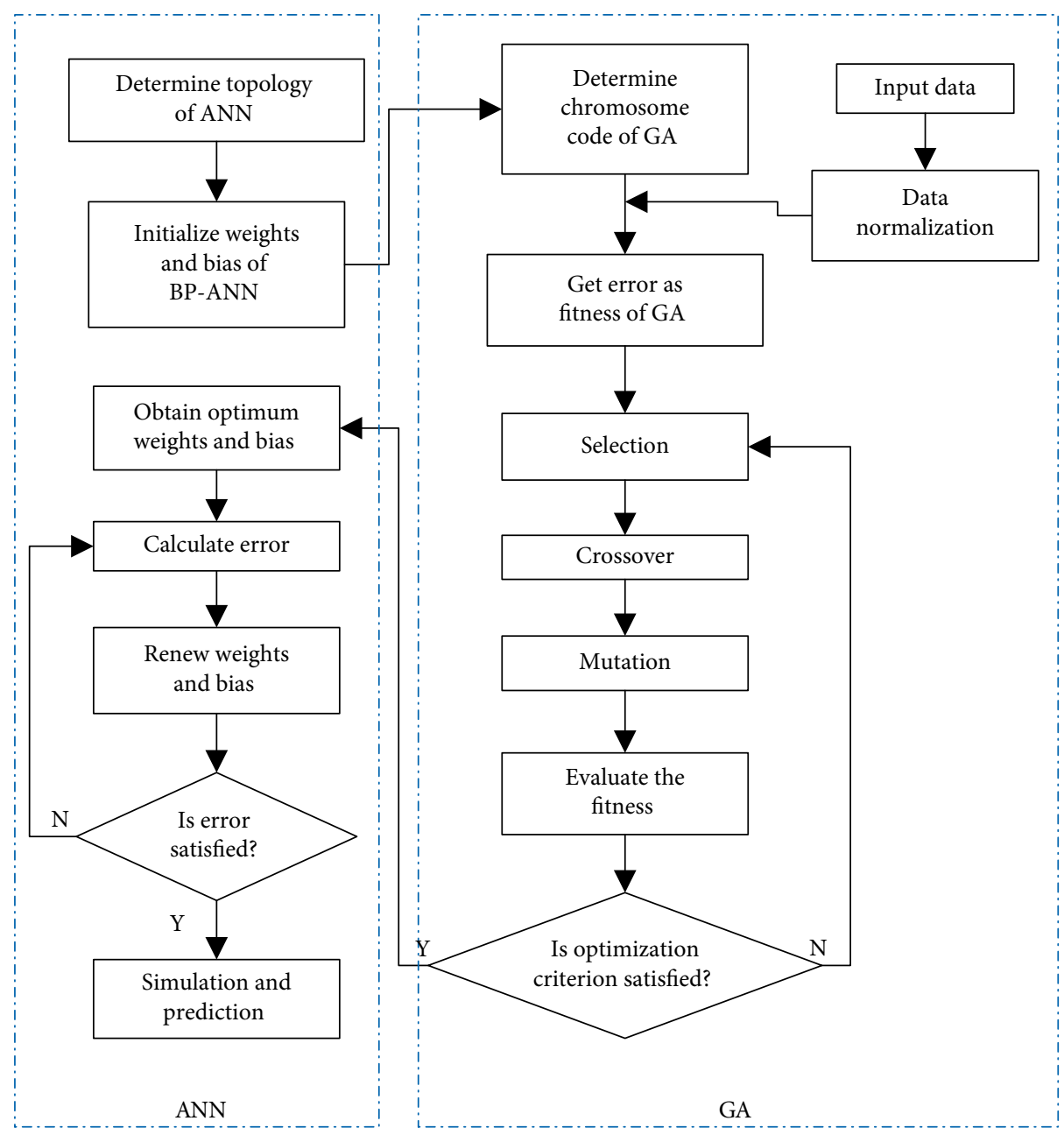

Figure 4: Flow chart of the hybrid model of ANN-GA.

\subsection{Hybrid Model of Artificial Neural Network and Genetic} Algorithm. GA was utilized to optimize the initial weights and bias for improving the prediction performance of ANN. The flow chart of ANN-GA is illustrated in Figure 4. The chromosome codes of GA consisted of the weights and bias of ANN. The mean square error between the experimental value and the prediction of ANN was used as the fitness function of GA. Then, genetic operations, such as selection, crossover, and mutation, were conducted to reproduce the new generation. As the new individuals replaced the parent individuals, the populations of GA were renewed. The abovementioned process repeated until the predefined generation number or the optimization criterion was satisfied.

Before training model, all the process parameter vectors and the observations in the simulations were normalized into the range $[-1,1]$ using the "mapmaxmin" function. All the calculations and optimizations were conducted under the environment of Matlab R2015b.

2.9. Statistical Analysis. To evaluate the performance of the different model for predicting SD, two statistic indicators including root mean square error (RMSE) and correlative coefficient $(\mathrm{R})$ were calculated. These statistical parameters are defined as the following:

$$
\begin{gathered}
\mathrm{RMSE}=\sqrt{\frac{\sum_{i=1}^{N}\left(y_{i}-x_{i}\right)^{2}}{N},} \\
\mathrm{R}=\frac{\sum_{i=1}^{N}\left(x_{i}-x_{\text {avg }}\right)\left(y_{i}-y_{\text {avg }}\right)}{\sqrt{\sum_{i=1}^{N}\left(x_{i}-\right)^{2} \sum_{i=1}^{N}\left(y_{i}-y_{\text {avg }}\right)^{2}}},
\end{gathered}
$$

where $x_{i}$ is the experimental value; $y_{i}$ is the corresponding prediction; $x_{\text {avg }}$ is the average of the experimental values; $y_{\text {avg }}$ is the average of the predictions; $N$ is the total number of simulations.

\section{Results and Discussion}

3.1. Simulation Results. The process of OWAIM includes a melt filling stage and a high-pressure filling stage. As shown in Figure 5(a), the mold cavity of the functional plastic part is filled with the high-temperature melt. During the short delay time, the outer layer of the melt is affected by the mold cavity, resulting in a decrease of temperature. Therefore, a thin melt layer with high viscosity is formed due to the heat conduction. Figure 5(b) demonstrates the result of high-pressure water 
TABle 3: ANOVA for Response Surface Quadratic Model.

\begin{tabular}{|c|c|c|c|c|c|c|}
\hline Source & Sum of squares & $\mathrm{df}$ & Mean square & $F$ value & $p$-value prob $>F$ & \\
\hline Model & 0.03 & 20 & $1.51 E-03$ & 4.61 & 0.0001 & Significant \\
\hline$X_{1}$ & $4.97 E-03$ & 1 & $4.97 E-03$ & 15.2 & 0.0005 & Significant \\
\hline$X_{2}$ & $2.13 E-04$ & 1 & $2.13 E-04$ & 0.65 & 0.4266 & \\
\hline$X_{3}$ & $7.35 E-03$ & 1 & $7.35 E-03$ & 22.5 & $<0.0001$ & Significant \\
\hline$X_{4}$ & $1.88 E-04$ & 1 & $1.88 E-04$ & 0.58 & 0.454 & \\
\hline$X_{5}$ & $4.43 E-03$ & 1 & $4.43 E-03$ & 13.55 & 0.0009 & Significant \\
\hline$X_{1} X_{2}$ & $5.25 E-05$ & 1 & $5.25 E-05$ & 0.16 & 0.6914 & \\
\hline$X_{1} X_{3}$ & $9.03 E-06$ & 1 & $9.03 E-06$ & 0.028 & 0.8691 & \\
\hline$X_{1} X_{4}$ & $3.71 E-04$ & 1 & $3.71 E-04$ & 1.14 & 0.2952 & \\
\hline$X_{1} X_{5}$ & $2.30 E-03$ & 1 & $2.30 E-03$ & 7.02 & 0.0129 & Significant \\
\hline$X_{2} X_{3}$ & $8.78 E-05$ & 1 & $8.78 E-05$ & 0.27 & 0.6082 & \\
\hline$X_{2} X_{4}$ & $5.53 E-04$ & 1 & $5.53 E-04$ & 1.69 & 0.2036 & \\
\hline$X_{2} X_{5}$ & $5.70 E-04$ & 1 & $5.70 E-04$ & 1.74 & 0.1971 & \\
\hline$X_{3} X_{4}$ & $2.13 E-03$ & 1 & $2.13 E-03$ & 6.51 & 0.0162 & Significant \\
\hline$X_{3} X_{5}$ & $9.57 E-04$ & 1 & $9.57 E-04$ & 2.93 & 0.0977 & \\
\hline$X_{4} X_{5}$ & $8.30 E-04$ & 1 & $8.30 E-04$ & 2.54 & 0.1218 & \\
\hline$X_{1}^{2}$ & $2.18 E-07$ & 1 & $2.18 E-07$ & $6.66 E-04$ & 0.9796 & \\
\hline$X_{2}^{2}$ & $3.16 E-04$ & 1 & $3.16 E-04$ & 0.97 & 0.3338 & \\
\hline$X_{3}^{2}$ & $1.50 E-04$ & 1 & $1.50 E-04$ & 0.46 & 0.503 & \\
\hline$X_{4}^{2}$ & $3.57 E-05$ & 1 & $3.57 E-05$ & 0.11 & 0.7435 & \\
\hline$X_{5}^{2}$ & $1.02 E-07$ & 1 & $1.02 E-07$ & $3.13 E-04$ & 0.986 & \\
\hline Residual & $9.48 E-03$ & 29 & $3.27 E-04$ & & & \\
\hline Lack of fit & $9.48 E-03$ & 22 & $4.31 E-04$ & & & \\
\hline Pure error & 0 & 7 & 0 & & & \\
\hline Cor total & 0.04 & 49 & & & & \\
\hline
\end{tabular}

TABLE 4: Optimal weights and bias for ANN-GA model.

\begin{tabular}{|c|c|c|c|c|c|c|c|}
\hline \multicolumn{6}{|c|}{ Hidden layer } & \multicolumn{2}{|c|}{ Output layer } \\
\hline & & Weights & & & Bias & Weights & Bias \\
\hline-0.045 & 0.411 & -0.219 & 0.327 & -0.389 & -0.209 & 0.307 & -0.243 \\
\hline 0.426 & 0.463 & 0.239 & -0.256 & -0.302 & -0.058 & -0.240 & \\
\hline 0.358 & 0.336 & -0.062 & 0.349 & -0.042 & -0.381 & 0.065 & \\
\hline 0.419 & -0.104 & 0.147 & 0.428 & -0.221 & -0.220 & -0.480 & \\
\hline-0.007 & 0.348 & -0.322 & -0.248 & 0.489 & 0.188 & -0.187 & \\
\hline 0.229 & 0.316 & 0.388 & 0.454 & 0.258 & 0.113 & -0.141 & \\
\hline 0.439 & -0.174 & -0.413 & -0.289 & 0.171 & -0.002 & -0.411 & \\
\hline 0.414 & 0.471 & -0.374 & 0.078 & 0.055 & 0.286 & 0.075 & \\
\hline-0.166 & 0.453 & 0.077 & 0.004 & 0.491 & -0.454 & 0.002 & \\
\hline 0.471 & 0.452 & 0.362 & -0.397 & -0.316 & -0.403 & 0.285 & \\
\hline-0.074 & -0.175 & -0.020 & 0.394 & 0.271 & 0.035 & -0.088 & \\
\hline
\end{tabular}

penetration. After the delay time, water is injected into the mold cavity and penetrates along the core with the least flow resistance. The melt is pushed forward to form a plastic part with a hollow cross-section.

3.2. Multiple Regression and Analysis of Variance. Based on the CCD, the results of the experiments are listed in Table 2. A second-order polynomial equation depicting the relationship between five independent process parameters and SD was determined.

$$
\begin{aligned}
\mathrm{SD}= & 0.15+0.012 X_{1}-0.0025 X_{2}-0.015 X_{3}+0.0024 X_{4} \\
& +0.011 X_{5}+0.0013 X_{1} X_{2}+0.0005 X_{1} X_{3}-0.0034 X_{1} X_{4} \\
& -0.0084 X_{1} X_{5}+0.0017 X_{2} X_{3}-0.0042 X_{2} X_{4}+0.0042 X_{2} X_{5} \\
& -0.0082 X_{3} X_{4}+0.0055 X_{3} X_{5}-0.0051 X_{4} X_{5}+0.0003 X_{1}^{2} \\
& +0.011 X_{2}^{2}+0.0078 X_{3}^{2}+0.0038 X_{4}^{2}-0.0002 X_{5}^{2} .
\end{aligned}
$$


(a)

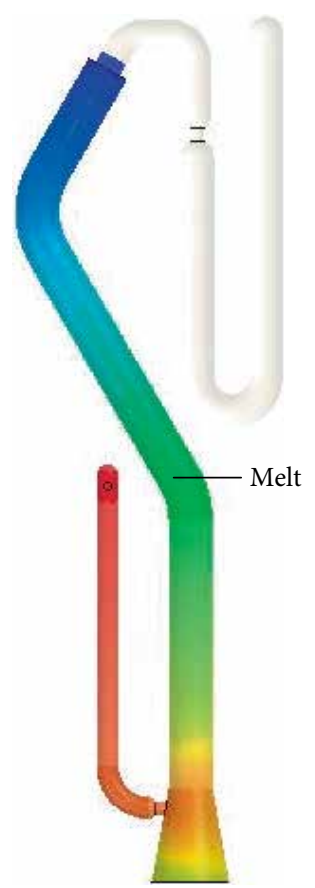

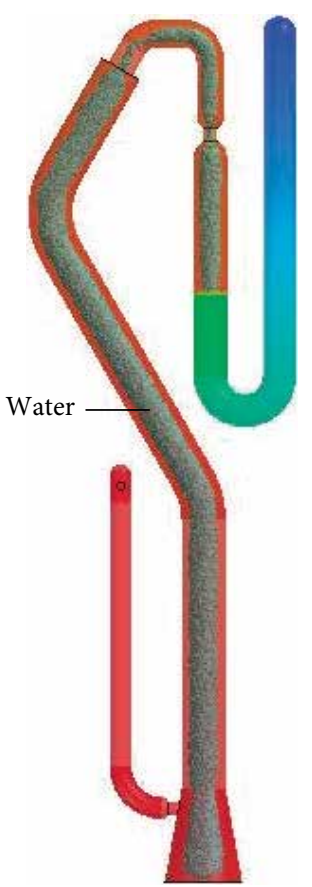

(b)
FIGURE 5: Simulation result and (a) melt filling stage, (b) water filling stage.

The adequacy and fitness of the quadratic model were further studied by ANOVA (Table 3). $p$-value $<0.05$ is a criterion for judgment about the significance of each term, and shows significant variables at $95 \%$ confidence level. The regression model ( $F=4.61$ and $p=0.0001)$ was statistically significant, which indicates that there was a good fit between the experimental data and predicted data obtained by RSM. As listed in Table 3, the linear coefficients of melt temperature, delay time, and water temperature were noted to be significant with the $F$ - and $p$-value of $(F=15.2, p=0.0005),(F=22.5, p<0.0001)$ and $(F=13.55, p=0.0009)$, respectively; the interaction between melt temperature and water temperature seemed to be predominant with $F$ - and $p$-value of $(F=7.02, p=0.0129)$, which was followed by the interaction between delay time and water pressure $(F=6.51, p=0.0162)$; all quadratic coefficients were insignificant.

3.3. Initial Weights and Bias of ANN-GA. The optimization of the initial weights and bias of ANN was implemented using GA. 37 of the 43 data sets were used to train the ANN-GA, and the rest were used to test this model. The mean square error between the predicted values and experimental values was used as a fitness function. As shown in Figure 6, during the training process, the fitness value firstly decreased sharply and then ran steadily after the 150th generation. The minimum fitness value was $4.3 E-4$ after the preset generations, which meant that the predictions were in good agreement with the targets. The initial weights and bias (Table 4) were fixed and assigned to ANN-GA model.

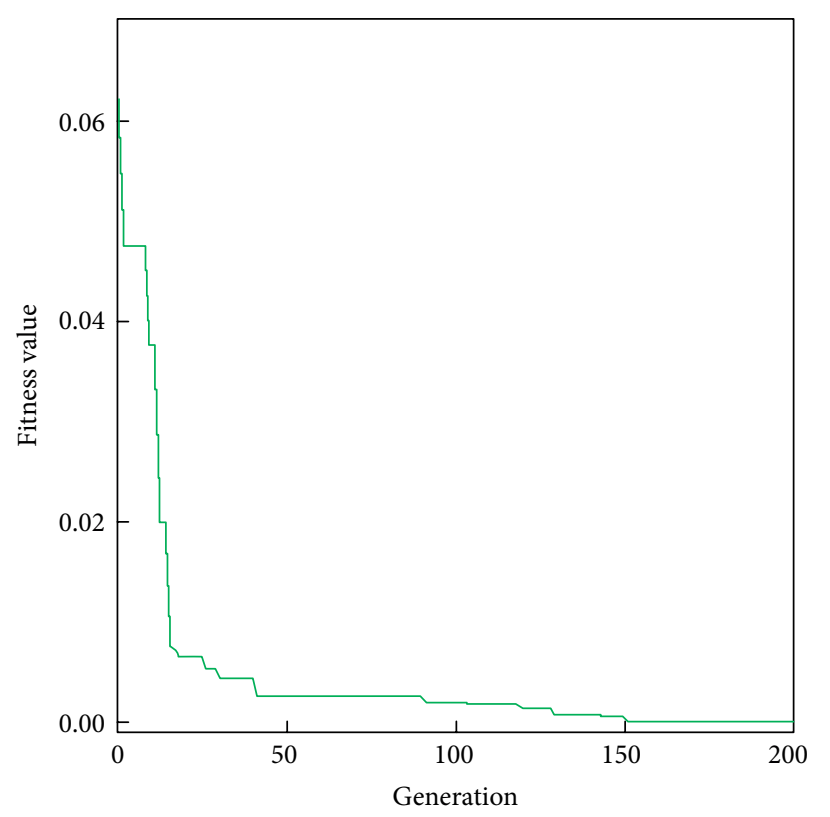

FIGURE 6: Evolution of fitness value for optimizing ANN-GA model.

of the experimental values to predicted values obtained by three models (RSM, ANN, and ANN-GA). The linear coefficient of the fitting line for RSM, ANN, and ANNGA models were $0.8147,0.9839$, and 1.001 , respectively. The data points of RSM were scattered on both sides of the fitting line, while the data points of ANN and ANNGA were very close to the fitting line. Moreover, the effectiveness of RSM, ANN, and ANN-GA models was statistically evaluated in terms of RMSE and $R$ values between the experimental values and predicted values. Table 5 gives the statistical parameters of RMSE and R for RSM, ANN, and ANN-GA models, respectively. In general, the closeness of the RMSE value to zero and the $R$ value to unity represents more accuracy of response predicted by three models. Through the analysis abovementioned, it was concluded that the three models (RAM, ANN, and ANN-GA) could well map the relationship between the independent process parameters and SD, and therefore can provide predictions with acceptable accuracy for unseen data sets; the best performance of prediction was ANN-GA followed by ANN and RSM, which indicates that the ANNGA model has the strongest ability to be generalized. Hence, the ANN-GA model was selected as the final prediction model in the optimization process.

3.5. Optimization Result of ANN-GA and Validation. The optimization of RWTU was carried out with the principle "the smaller, the better". The expression based of ANN-GA for calculating SD was used as the fitness function of GA. The evolution of this optimization process was recorded in Figure 8 . The lines representing fitness value run steadily after the 40th generation. The optimized process parameters of melt temperature $=219^{\circ} \mathrm{C}$, mold temperature $=59.8^{\circ} \mathrm{C}$, water injection delay time $=5 \mathrm{~s}$, water pressure $=10.2 \mathrm{MPa}$ and water temperature $=20^{\circ} \mathrm{C}$ were considered. With the optimized 


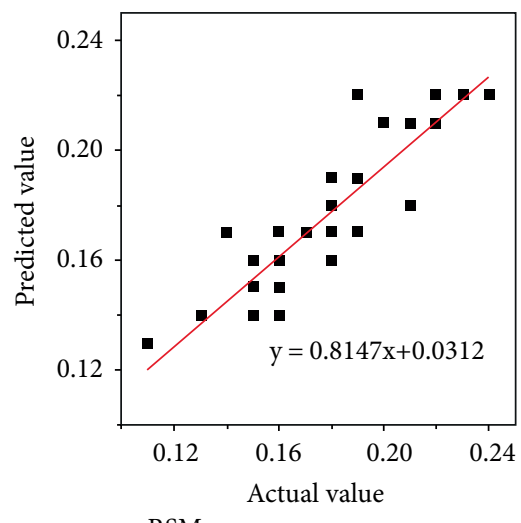

- RSM

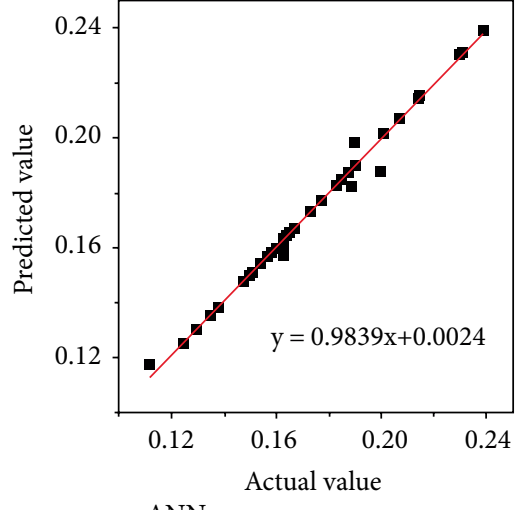

(b)

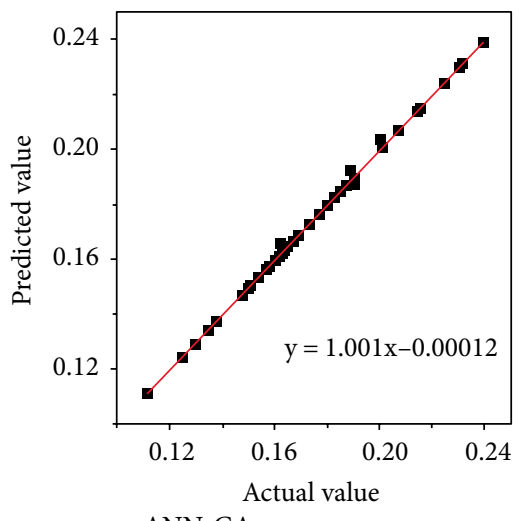

- ANN-GA

(a)

FIgURE 7: Predicted values versus the actual values, (a) RSM, (b) ANN, (c) ANN-GA.

TABLE 5: Statistical parameters of RSM, ANN and ANN-GA model.

\begin{tabular}{lcc}
\hline Model & RMSE & R \\
\hline RSM & 0.0142 & 0.8731 \\
ANN & 0.0114 & 0.9397 \\
ANN-GA & 0.0010 & 0.9994 \\
\hline
\end{tabular}

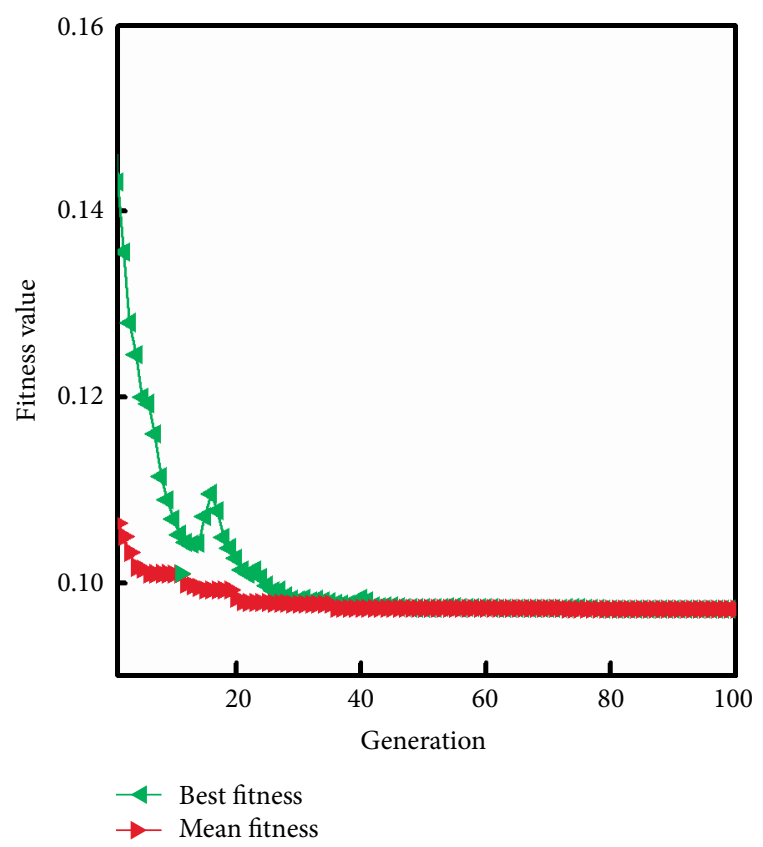

FIgURE 8: Evolution of fitness value in 100 generations.

process parameters, the SD predicted by ANN-GA model was 0.0972, which was smaller than any observation in simulation.

Using the optimal process parameters, a simulation experiment and real experiments were carried out to verify the optimization results. As shown in Figure 9, the value of SD obtained by the simulation experiment was 0.09 , which was slightly smaller than the predicted value of ANN-GA and smaller than any observation in the simulation experiments (Table 2). The

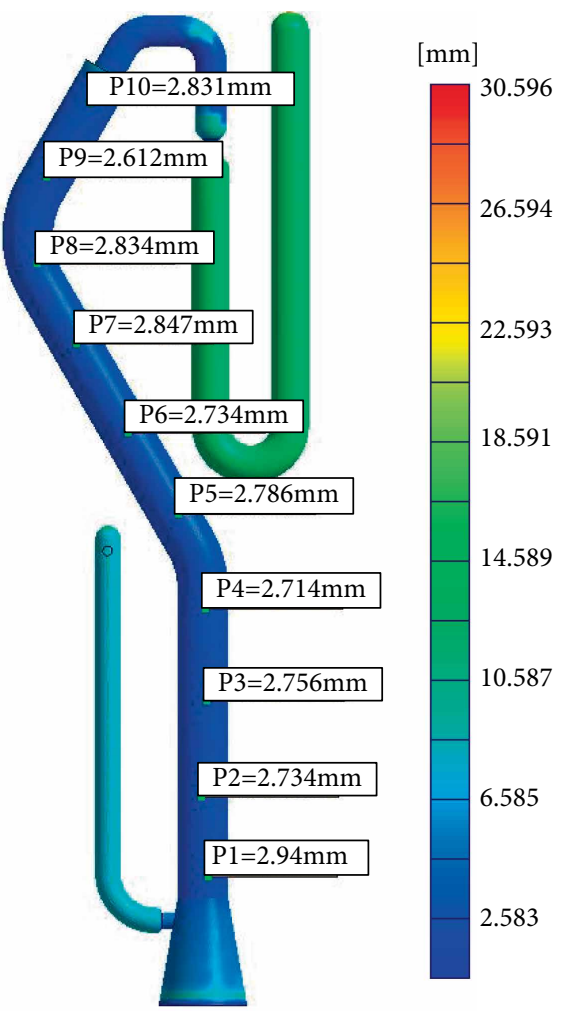

FIGURE 9: Simulation results with optimal process parameters.

results of the real experiments showed that the RWTU obtained by the optimal process parameters was improved.

\section{Conclusions}

The simulations of SFRC OWAIM were carried out, and the SD was used to characterize the RWTU of plastic parts with hollow cross-sections. RSM-CCD with five independent variables (melt temperature, mold temperature, delay time, water pressure, and water temperature) at three levels and ANN-GA with one hidden layer were employed to construct models for predicting SD. The influences of five process parameters on RWTU were studied using ANOVA, indicating melt temperature, delay time, and 
water temperature were predominant. The prediction abilities of three models (RSM, ANN, and ANN-GA) were compared through some statistical criteria and the results demonstrated that the ANN-GA model had the best performance followed by ANN and RSM. The optimization of RWTU was implemented using ANN-GA as a fitness function and got the minimum SD 0.097 which was smaller than any other experimental result. It is noteworthy that our interesting findings will be provided new solutions for the subsequent optimization of warpage and shrinkage of plastic parts in SFRC OWAIM.

\section{Data Availability}

The data used to support the findings of this study are available from the corresponding author upon request.

\section{Conflicts of Interest}

The authors declare that they have no conflicts of interest.

\section{Acknowledgments}

The work in this paper is funded by the National Natural Science Foundation of China (Grant nos. 21664002 and 51563010) and the Science and Technology Project of Education Department of Jiangxi, China (Grant no. GJJ150609). The authors would like to express their sincere gratitude to those who made comments on the changes proposed in this article.

\section{References}

[1] J. G. Yang, X. H. Zhou, and Q. Niu, "Model and simulation of water penetration in water-assisted injection molding," The International Journal of Advanced Manufacturing Technology, vol. 67, no. 1-4, pp. 367-375, 2013.

[2] L. Li, Y. Peng, and W. Wei, "Recent advances on fluid assisted injection molding technique," Recent Patents on Mechanical Engineering, vol. 7, no. 1, pp. 82-91, 2014.

[3] T.-Q. Kuang, K. Zhou, L.-X. Wu, G.-F. Zhou, and L.-S. Turng, "Experimental study on the penetration interfaces of pipes with different cross-sections in overflow water-assisted coinjection molding," Journal of Applied Polymer Science, vol. 133, no. 1, 2016.

[4] T. Kuang, C. Yu, B. Xu, and L. S. Turng, "Experimental study of penetration interfaces in the overflow fluid-assisted co-injection molding process," Journal of Polymer Engineering, vol. 36, no. 2, pp. 139-148, 2016.

[5] P. Olley, L. Mulvaney-Johnson, and P. D. Coates, "Nonisothermal experimental and simulation study of residual wall thickness in gas assisted injection moulding," Plastics, Rubber and Composites, vol. 35, no. 2, pp. 47-58, 2006.

[6] K.-Y. Lin, F.-A. Chang, and S.-J. Liu, "Using differential mold temperatures to improve the residual wall thickness uniformity around curved sections of fluid assisted injection molded tubes," International Communications in Heat and Mass Transfer, vol. 36, no. 5, pp. 491-497, 2009.
[7] H. Park and B. Rhee, "Effects of the viscosity and thermal property of fluids on the residual wall thickness and concentricity of the hollow products in fluid-assisted injection molding," The International Journal of Advanced Manufacturing Technology, vol. 86, no. 9-12, pp. 3255-3265, 2016.

[8] H.-P. Park, B.-S. Cha, S.-B. Park et al., "A study on the void formation in residual wall thickness of fluid-assisted injection molding parts," Advances in Materials Science and Engineering, vol. 2014, Article ID 238251, 6 pages, 2014.

[9] T. Pudpong, P. Buahom, S. Areerat, W. Rungseesantivanon, I. Satoh, and T. Saito, "The effects of processing parameters on the residual wall thickness distribution at the sharp angle corner of water assisted injection molded parts," International Polymer Processing, vol. 28, no. 5, pp. 528-540, 2013.

[10] H.-X. Huang, R.-H. Zhou, and C. Yang, "Fiber orientation propelled by high-pressure water penetration in water-assisted injection molded fiber-reinforced thermoplastics part," Journal of Composite Materials, vol. 47, no. 2, pp. 183-190, 2013.

[11] S.-J. Liu, M.-J. Lin, and Y.-C. Wu, "An experimental study of the water-assisted injection molding of glass fiber filled polybutylene-terephthalate (PBT) composites," Composites Science and Technology, vol. 67, no. 7-8, pp. 1415-1424, 2007.

[12] C. Shen, L. Wang, and Q. Li, “Optimization of injection molding process parameters using combination of artificial neural network and genetic algorithm method," Journal of Materials Processing Technology, vol. 183, no. 2-3, pp. 412-418, 2007.

[13] P. Pan, W. Jin, X. Li et al., "Optimization of multiplex quantitative polymerase chain reaction based on response surface methodology and an artificial neural network-genetic algorithm approach," PLoS ONE, vol. 13, no. 7, Article ID e0200962, 2018.

[14] S. Jacob and R. Banerjee, "Modeling and optimization of anaerobic codigestion of potato waste and aquatic weed by response surface methodology and artificial neural network coupled genetic algorithm," Bioresource Technology, vol. 214, pp. 386-395, 2016.

[15] N. Salim, A. Santhiagu, and K. Joji, "Process modeling and optimization of high yielding L-methioninase from a newly isolated Trichoderma harzianum using response surface methodology and artificial neural network coupled genetic algorithm," Biocatalysis and Agricultural Biotechnology, vol. 17, pp. 299-308, 2019.

[16] J.-X. Yang and G.-B. Hong, "Optimized extraction for active compounds in Glossogyne tenuifolia using response surface methodology," Journal of Food Measurement and Characterization, vol. 13, no. 1, pp. 663-676, 2019.

[17] A. A. B. M. Zin and M. Moradi, "An experimental investigation of price elasticity in electricity markets using a response surface methodology," Energy Efficiency, vol. 12, no. 3, pp. 667-680, 2019.

[18] Y. Horie, T. Yoshio, K. Aoyama et al., "Diagnostic outcomes of esophageal cancer by artificial intelligence using convolutional neural networks," Gastrointestinal Endoscopy, vol. 89, no. 1, pp. 25-32, 2019.

[19] A. Dehghanbanadaki, M. A. Sotoudeh, I. Golpazir, A. Keshtkarbanaeemoghadam, and M. Ilbeigi, "Prediction of geotechnical properties of treated fibrous peat by artificial neural networks," Bulletin of Engineering Geology and the Environment, vol. 78, no. 3, pp. 1345-1358, 2018.

[20] D. Wang, M. Zhang, Z. Li et al., "System impairment compensation in coherent optical communications by using 
a bio-inspired detector based on artificial neural network and genetic algorithm," Optics Communications, vol. 399, pp. 1-12, 2017.

[21] S. Ghanavati Nasab, A. Semnani, A. Teimouri, H. Kahkesh, T. Momeni Isfahani, and S. Habibollahi, "Removal of congo red from aqueous solution by hydroxyapatite nanoparticles loaded on zein as an efficient and green adsorbent: response surface methodology and artificial neural network-genetic algorithm," Journal of Polymers and the Environment, vol. 26, no. 9, pp. 3677-3697, 2018.

[22] S. Bahrami, F. Doulati Ardejani, and E. Baafi, "Application of artificial neural network coupled with genetic algorithm and simulated annealing to solve groundwater inflow problem to an advancing open pit mine," Journal of Hydrology, vol. 536, pp. 471-484, 2016. 